PoS $\quad \begin{aligned} & \text { PROCEEDINGS } \\ & \text { OF SCIENCE }\end{aligned}$

\title{
Toward a new indirect approach for nuclear astrophysics. A case study
}

\section{La Cognata*}

Laboratori Nazionali del Sud - Istituto Nazionale di Fisica Nucleare, Catania, Italy

E-mail: lacognataelns.infn.it

\section{G.G. Kiss}

ATOMKI, Debrecen, Hungary

O. Trippella

University of Perugia and Sezione di Perugia - Istituto Nazionale di Fisica Nucleare, Perugia, Italy

\section{Spitaleri}

University of Catania and Laboratori Nazionali del Sud - Istituto Nazionale di Fisica Nucleare,

Catania, Italy

\section{A.M. Mukhamedzhanov}

Cyclotron Institute, Texas A\&M University, College Station, Texas, USA 
Fusion reactions play a key role in astrophysics, as they are responsible of energy production and nucleosynthesis. The characteristic temperatures range from $10^{9} \mathrm{~K}$ in hot explosive scenarios to $10^{6}-10^{7} \mathrm{~K}$ in quiescent burning in low-mass stars. Therefore, the relevant cross sections have to be known at energies smaller than $\sim 1 \mathrm{MeV}$. However, the Coulomb barrier and the electron screening effect make it very difficult to provide accurate cross sections. The presence of sub threshold resonances might radically alter the expected trend of the extrapolated cross section, leading to systematic errors. The ${ }^{13} \mathrm{C}(\alpha, n){ }^{16} \mathrm{O}$ reaction is an example of reactions characterized by strong sub threshold resonances. ${ }^{13} \mathrm{C}(\alpha, n){ }^{16} \mathrm{O}$ is the neutron source of the main component of the s-process, responsible of the production of most nuclei in the mass range $90<A<204$. It is active inside the helium-burning shell in AGB stars, at temperatures $\sim 10^{8} \mathrm{~K}$, corresponding to an energy interval of $140-230 \mathrm{keV}$. Though it plays a crucial role, no direct measurements exist inside the $140-230 \mathrm{keV}$ range. Extrapolations through the R-matrix and indirect techniques such as the ANC yield inconsistent results. The discrepancy amounts to a factor of 3 or more. Therefore, we have applied the THM to the ${ }^{13} \mathrm{C}(\alpha, n){ }^{16} \mathrm{O}$ reaction. For the first time, the ANC for the $6.356 \mathrm{MeV}$ level has been deduced through the THM, allowing to attain an unprecedented accuracy in the ${ }^{13} \mathrm{C}(\alpha, n){ }^{16} \mathrm{O}$ study. This innovative approach, merging together two well establish indirect techniques, can find application in the study of a number of resonance reactions at deep sub Coulomb and even negative energies, where direct measurements are impossible.

XIII Nuclei in the Cosmos,

7-11 July, 2014

Debrecen, Hungary

${ }^{*}$ Speaker. 


\section{The THM for resonant reactions}

Because of the problems direct measurements have shown in the years (compare [1,2] for a detailed analysis), indirect techniques have been developed attempting to bypass them and attain the astrophysical factor at low energies. Indeed, the Coulomb barrier suppresses the cross section to values lower than picobarn, hardly attainable using present day facilities. Moreover, since interaction energies are of the same order of atomic energies, the electron screening effect makes the bare nucleus cross section, that is the input parameter for astrophysics, unaccessible but through extrapolation even in those cases where astrophysical energies have been achieved.

For instance, the Trojan Horse Method (THM) [3, 4] is a valid technique to get information on the $S(E)$ factor at astrophysical energies in the case of reactions having charged particles or neutrons in the exit channel, with no Coulomb suppression neither electron screening. In the case of radiative capture reactions, the Asymptotic Normalization Coefficient (ANC) [5] measurement has allowed us to obtain the zero-energy $S(E)$ factor with very high accuracy. The Coulomb dissociation (CD) was also developed [6] to establish the low energy behavior of charged-particle induced reactions having a $\gamma$-ray in the exit channel.

In more recent years, A.M. Mukhamedzhanov has strongly improved the theoretical background of the THM [4], introducing the approach called generalized R-matrix formalism, allowing for the analysis of multi-resonance reactions [7] with unprecedented accuracy.

In the modified R-matrix framework, assuming for simplicity that the $A(x, c) C$ reaction proceeds via isolated non-interfering resonances, so that the two-level, one-channel modified R-matrix formula applies, the cross section of the THM reaction can be written as [7, 8]:

$$
\begin{array}{r}
\frac{\mathrm{d}^{2} \sigma}{\mathrm{d} E_{x A} \mathrm{~d} \Omega_{s}}=\mathrm{NF} \sum_{i}\left(2 \mathrm{~J}_{i}+1\right) \\
\times\left|\sqrt{\frac{\mathrm{k}_{\mathrm{f}}\left(E_{x A}\right)}{\mu_{c C}}} \frac{\sqrt{2 P_{l_{i}}\left(k_{c C} R_{c C}\right)} M_{i}\left(p_{x A} R_{x A}\right) \gamma_{c C}^{i} \gamma_{x A}^{i}}{D_{i}\left(E_{x A}\right)}\right|^{2},
\end{array}
$$

in the PWIA approach. Here, NF is a normalization factor, $\mathrm{k}_{f}\left(E_{x A}\right)=\sqrt{2 \mu_{c C}\left(E_{x A}+Q\right)} / \hbar(\mathrm{Q}$ is the Q-value of the $A(x, c) C$ reaction, $E_{x A}$ the $x-A$-relative energy), $R_{x A}$ and $R_{c C}$ the channel radii for the entrance and exit channels, respectively,

$$
M_{\tau}\left(p_{x A} R_{x A}\right)=\left[\left(B_{x A}-1\right) j_{l_{\tau}}(\rho)-\rho \frac{\partial j_{l_{\tau}}(\rho)}{\partial \rho}\right]_{\rho=p_{x A} R_{x A}}
$$

where $j_{l_{i}}(\rho)$ is the spherical Bessel function, $p_{x A}=\sqrt{2 \mu_{x A}\left(E_{x A}+B_{x s}\right)} / \hbar\left(B_{x s}\right.$ the binding energy of the $a=x \oplus s$ system, the so called Trojan-horse nucleus), and $B_{x A}^{i}$ an arbitrary boundary condition. Adopting the natural boundary condition, the Thomas approximation can be used to yield the observable resonance parameters $[4,9,10,11] . D_{i}\left(E_{x A}\right)$ is the standard R-matrix denominator in the case of two-level, one-channel R-matrix formulas, containing shift and penetration functions besides the boundary conditions [8]. By introducing the level matrix $\mathbf{A}[4,7,9]$, a similar equation can be obtained in the two-level, two-channel case, which is needed to account for interference effects. 
In general, a different inference pattern is got as a different mechanism for the population of the resonance states is acting in the THM approach, the $M$-factor in Eq. (1.2) entering the Rmatrix expression in the place of the entrance-channel penetration factor. On the other hand, in the modified R-matrix approach, the same reduced widths appear as in the OES S(E)-factor, the only difference being the absence of any Coulomb or centrifugal penetration factor in the entrance channel. From the fitting of the experimental THM cross section through Eq. (1.1) the reduced widths $\gamma_{\tau}^{i}$ can be obtained and used to deduce the OES astrophysical factor, which turns out to be not affected by the electron screening or Coulomb barrier suppression. As in the narrow resonance case, normalization is achieved by extending the indirect measurement to an energy region where directly measured resonances are available and scaling the deduced $\gamma$-widths to match the values in the literature.

By choosing a suitable beam energy and TH nucleus, negative energies can be attained in $A-x$ even if the energy of projectile $A$ exceeds the Coulomb barrier in the initial channel, $A+a$, of the $\mathrm{TH}$ reaction, owing to the binding energy $\varepsilon_{s x}$. The reason why negative energies can the explored can be understood if energy and momentum conservation in the three-ray vertex, $a \rightarrow s+x$, and in the four-ray vertex, $A+x \rightarrow C+c$, of the THM pole diagram [12] are examined. Denoted by $\varepsilon_{i j}$, $E_{i j}$ and $p_{i j}$ the binding energy, the relative energy and the relative momentum of particles $i$ and $j$, respectively, in [13] we concluded that:

$$
E_{A x}=\frac{p_{A x}^{2}}{2 \mu_{A x}}-\frac{p_{s x}^{2}}{2 \mu_{s x}}-\varepsilon_{s x},
$$

where $\mu_{i j}$ is the reduced mass of the $i-j$ system. Assuming that the $x-s$ relative motion takes place in s-wave (like the $p-n$ motion inside deuteron or the $d-\alpha$ motion inside ${ }^{6} \mathrm{Li}$ ), in $\mathrm{QF}$ kinematics $p_{s x}=0$ and

$$
E_{A x}=\frac{p_{A x}^{2}}{2 \mu_{A x}}-\varepsilon_{s x}
$$

\section{Merging THM and ANC}

When dealing with sub threshold resonances, the equations above have to be modified as for bound states at negative energies penetrability is zero, but the shift function can still be defined as the logarithmic derivative of the Whittaker function [10].

The R-matrix eigenfunction $w(r)$ is proportional to $\phi(r)$, the true (single-channel) bound state, inside the R-matrix radius $a$ : $\phi(r)=A w(r)$, where $A^{2}=1-\int_{a}^{\infty}|\phi(r)|^{2} d r$ reflects the different normalization requirements. Outside the R-matrix radius, the Whittaker function $W(r)$ describes the asymptotic behavior of the bound state wave function of two charged particles, the asymptotic normalization coefficient (ANC) defining the amplitude of its tail, namely, the ANC value $C$ is the coefficient in $\phi(r)=C W(r)$ for the Whittaker function [14, 15]. The reduced width is therefore [10]:

$$
\gamma^{2}=\frac{\hbar^{2}}{2 \mu a} \frac{C^{2} W(a)^{2}}{1-C^{2} \int_{a}^{\infty}|W(r)|^{2} d r} .
$$

For deeply bound states $A \sim 1$, and then we will have the proportionality $\gamma^{2} \propto C^{2}$, but the integral in the denominator may become important for weakly bound or halo states $[10,15]$. Therefore, 
from the THM measurement of a sub-threshold state, yielding the reduced widths $\gamma$, the ANC can be deduced, clearly disclosing the deep connection of the two indirect approaches [12, 16].

However, the THM approach to the extraction of the ANC presents some advantages, which are discussed in details in [12]. In the standard approach, angular distributions of transfer reactions are usually measured and fitted in different theoretical frameworks, to evaluate the sensitivity of the deduced ANC on the optical potentials used in calculations. As shown in previous sections, ambiguities on the reaction mechanism, transfer vs. compound nucleus, for instance, might arise introducing systematic errors of poorly known size. Moreover, background reactions might determine an inaccurate estimate of the true counting rate if incorrectly subtracted. By contrast, three-body kinematics make it possible a number of kinetic tests leading to an unambiguous identification of the reaction channel, allowing us to separate the $A(a, c C) s$ channel from background reactions [3] and to single out the requested branch (e.g., the population of particle $C$ ground state). Necessary condition for the application of the THM is the evaluation of the presence of the QF mechanism, which has to be disentangled from other competing reaction mechanisms. Because of the clear signature of the QF process, this reaction mechanism can be unambiguously singled out from the $A(a, c C) s$ reaction yield.

\section{The ${ }^{13} \mathrm{C}(\alpha, n){ }^{16} \mathrm{O}$ reaction: a case study}

The ${ }^{13} \mathrm{C}(\alpha, n){ }^{16} \mathrm{O}$ reaction is considered as the main neutron supply providing for the neutron flux necessary to build up heavy elements from iron-peak seed nuclei (s-process). In the He-burning shell of AGB stars, where the main component of the $s$-process is produced, temperatures vary between $10^{7}$ and $0.9 \times 10^{8} \mathrm{~K}$ during the time that the $\mathrm{H}$-shell is the major nuclear source in the star, while temperatures can reach to $3 \times 10^{8} \mathrm{~K}$ during the He-burning phase. At $0.9 \times 10^{8} \mathrm{~K}$, the energy range where the ${ }^{13} \mathrm{C}(\alpha, n){ }^{16} \mathrm{O}$ reaction is most effective, the so-called Gamow window [2], is within $\sim 140-230 \mathrm{keV}$. At such small energies the Coulomb barrier exponentially reduces the fusion cross section $\sigma$ leading to values as small as $10^{-11}$ barn at the Gamow energies. Such small values are very difficult to measure as the signal-to-noise ratio rapidly approaches zero. Extrapolation, supported by nuclear theory such as the R-matrix, has been used to determine the cross section values at astrophysical energies. In the ${ }^{13} \mathrm{C}(\alpha, n){ }^{16} \mathrm{O}$ case, extrapolation is complicated by the occurrence of a sub threshold resonance at $-3 \mathrm{keV}$ due to the $6.356 \mathrm{MeV}$ level of ${ }^{17} \mathrm{O}$, causing an increase of the astrophysical factor as $E$ draws closer to zero.

For this reason we have performed a new measurement of the ${ }^{13} \mathrm{C}(\alpha, n){ }^{16} \mathrm{O}$ at astrophysical energies, using the Trojan Horse Method (THM). The modified R-matrix approach has been used to fit the THM data and deduce the resonance parameters $[12,16]$. In the present work, normalization is performed in the $0.5-1.2 \mathrm{MeV}$ energy window, since four resonances are present in this interval, whose parameters are well known [17]. Moreover, in this region the high energy tail of the $3 \mathrm{keV}$ resonance has a vanishingly small contribution, as the electron screening effect. The THM approach allowed us to extract the Coulomb-modified ANC $\tilde{C}_{\alpha^{17} \mathrm{C}}^{17} \mathrm{O}\left(2^{+}\right)$of the $-3 \mathrm{keV}$ resonance, from the HOES R-matrix fitting of the THM data. This is the first time that THM is used to extract the ANC of a sub threshold resonance. In detail, following the discussion in [15, 16], we obtained $\left(\tilde{C}_{\alpha^{13} \mathrm{C}}^{17} \mathrm{O}\left(1 / 2^{+}\right)\right)^{2}=7.7 \pm 0.3_{\text {stat }}{ }_{-1.5}^{+1.6}$ norm $\mathrm{fm}^{-1}$ in agreement, within the uncertainties, with our preliminary value $6.7_{-0.6}^{+0.9} \mathrm{fm}^{-1}$. The comparison with the ANC's for previous measurements 
and, where not available, the spectroscopic factors $S_{\alpha}$ for the $6.356 \mathrm{keV}{ }^{17} \mathrm{O}$ level indicates that our result is well consistent with the ANC deduced from the spectroscopic factor measurement by [18], and probably with the result of [19] once the increase of $S_{\alpha}$ for increasing beam energy observed by [18] is accounted for. Using the reduced widths deduced through the Modified R-matrix approach, the ${ }^{13} \mathrm{C}(\alpha, n){ }^{16} \mathrm{O} S$-factor at $100 \mathrm{keV}$ of $4.0 \pm 0.7 \times 10^{6} \mathrm{MeVb}$. This result agrees quite well with the largest $S(100 \mathrm{keV})$, based on extrapolations of direct data or ANC or spectroscopic factors from transfer reactions, with an improved accuracy due to a reduced systematic uncertainty (check Refs. $[12,16]$ for more details). As a consequence, the reaction rate deduced from the THM $S$-factor is in agreement with the most results in the literature at $\sim 10^{8} \mathrm{~K}$, with enhanced accuracy thanks to this innovative approach. The possible astrophysical consequences of the present work are currently under investigation [12].

\section{Acknowledgements}

The work was supported in part by the Italian MIUR under grant No. RBFR082838

\section{References}

[1] C. E. Rolfs and W. S. Rodney, Cauldrons in the cosmos (University of Chicago Press, Chicago, IL, 1988)

[2] C. Iliadis, Nuclear Physics of Stars (Wiley-VCH Verlag, Weinheim, 2007)

[3] C. Spitaleri et al., Physics of Atomic Nuclei 74, 1725 (2011)

[4] A. M. Mukhamedzhanov et al., J. Phys. G 35, 014016 (2008)

[5] A. M. Mukhamedzhanov et al., Phys. Rev. C 56, 1302 (1997)

[6] G. Baur \& H. Rebel, J. Phys. G.: Nucl. Part. Phys. 20, 1 (1994)

[7] A. M. Mukhamedzhanov, Phys. Rev. C 84, 044616 (2011)

[8] M. La Cognata et al., Astrophys. J. 739, L54 (2011)

[9] M. La Cognata, C. Spitaleri, and A. M. Mukhamedzhanov, Astrophys. J. 723, 1512 (2010)

[10] I. J. Thompson \& F.M. Nunes, Nuclear Reactions for Astrophysics: Principles, Calculation and Applications of Low-Energy Reactions (Cambridge University Press, Cambridge, UK, 2009)

[11] R.G. Thomas, Phys. Rev. 81, 148 (1951)

[12] M. La Cognata et al., Astrophys. J. 777, 143 (2013)

[13] M. La Cognata et al., Phys. Rev. C 76, 065804 (2007)

[14] A.M. Mukhamedzhanov, N.K. Timofeyuk \& R.E. Tribble, Phys. Rev. C 51, 3472 (1995)

[15] A.M. Mukhamedzhanov \& R.E. Tribble, Phys. Rev. C 59, 3418 (1999)

[16] M. La Cognata et al., Phys. Rev. Lett. 109, 232701 (2012)

[17] M. Heil et al., Phys. Rev. C 78, 025803 (2008)

[18] M. G. Pellegriti et al., Phys. Rev. C 77, 042801 (2008)

[19] B. Guo et al., Astrophys. J. 756, 193 (2012) 